\title{
Sentido de comunidad y privacidad
}

\author{
Luis Gómez Jacinto, M. ${ }^{a}$ Isabel Hombrados Mendieta \\ Universidad de Málaga
}

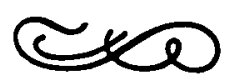

\section{Resumen}

Se realizaron dos investigaciones empíricas siguiendo una estrategia de observación pasiva con modelos causales; sobre dos muestras de 339 y 95 sujetos, respectivamente. El objetivo de las mismas es poner a prueba la hipótesis de la relación negativa entre privacidad y comunidad; así como estudiar la incidencia de algunas características psicológicas, sociales y ambientales sobre ambos conceptos. Los resultados ratifican la esperada relación negativa entre privacidad y comunidad. Se confirma la importancia del impacto sobre dichos fenómenos de las variables consideradas como determinantes, pero sorprende el efecto contrario al esperado de las variables relacionadas con la densidad de población, que fue inverso al que proponía nuestro modelo.

Palabras clave: privacidad, comunidad.

\section{Sense of privacy and sense of community}

\section{Abstract}

Two empirical studies were carried out following a strategy of passive observation with causal models; using two samples of 339 and 95 subjects respectively. The aim of these studies was to test the hypothesis of the negative relationship between privacy and community; likewise, to study the implications of some psychological, social, and environmental characteristics for both concepts. The results support the expected negative relationship between privacy and community. The importance of the impact on said phenomena of the variables regarded as determining is confirmed, but the opposite effect to that expected of the variables related to population density was found.

Key words: Privacy, community.

NOTA: El segundo de los estudios de investigación se realizó dentro del marco del curso de doctorado «Privacidad y regulación del contacto social», con la colaboración de los alumnos Marcos Martínez López, Inmaculada Pareja Tello, Africa Poloche Ferrero y José Luis Rioja García.

Dirección de los autores: Departamento de Psicología Social y de la Personalidad Campus. El Ejido. Universidad de Málaga. Málaga. 


\section{INTRODUCCION}

«Al principio no fue el verbo, sino la comunidad.» Bien podría haberse puesto esta frase en el pórtico del siglo que se inaugura con la recientemente conmemorada revolución francesa. Y hubiese sido una más de las muchas contradicciones y dualidades sobre las que sentó sus cimientos y construyó sus cien años; por que otro gran estallido, esta vez el de la primera gran guerra, sorprendió al hombre recortando «una parcela de la continuidad e infinitud del espacio» (Simmel, 1986, p. 31), haciéndola propia y estableciendo «una barrera interior entre la gente». Comunidad y privacidad, dos conceptos que polarizan buena parte de la historia occidental, de nuevo, en esta postrimería del siglo, que como en la anterior, destapan el frasco de nuestros deseos más utópicos y, porqué no decirlo, más contradictorios.

Pero privacidad y comunidad no son conceptos separados. Muy probablemente expresan aspectos diferentes de una única experiencia o sentimiento. $\mathrm{Si}$ la privacidad pertenece al universo del sí mismo y de la intimidad con otras personas; la comunidad gira en el mundo de la pertenencia al grupo, de la voluntad de estar entre los otros. Ambas son formas de la vida social: se tiene sentido de sí mismo en la medida en que se tiene sentido de los demás y viceversa (Esser y Greenbie, 1979).

No es mucho el interés que la psicología social muestra por ambos conceptos. Por lo que respecta a la privacidad hay que lamentarse con Kelvin (1973) del olvido a que los manuales y revistas de la disciplina lo han condenado. Tres razones aduce este autor para explicar esa desatención. En primer lugar, la palabra privacidad tiene en nuestra cultura unas connotaciones que la aproximan a lo sagrado y tabú. Su investigación científica etiqueta a los profanadores como social y moralmente «sospechosos». La segunda razón, derivada en parte de la anterior, es que la investigación empírica de la privacidad supone de hecho una invasión de la misma, distorsionando el fenómeno que se pretende observar por el mismo hecho de la observación. A esto nosotros añadiríamos el miedo a poner de manifiesto los atentados contra la vida privada que supone la mayoría de las investigaciones psicosociales (Kimmel, 1988). En definitiva, que nadie hurga en sus propias heridas. Y, por último, apunta este autor que la vaguedad definicional, la cercanía al concepto cotidiano, la variopinta terminología con que se dibuja el concepto, son un límite a su abordaje científico. Pero no parece que esta dificultad haya detenido a los psicólogos sociales en otras ocasiones; la libertad, el poder, el amor, la amistad, están rodeados de una niebla similar a la que rodea a la privacidad y ello no ha sido óbice para que pasasen, incluso, por el tamiz del laboratorio. Fue el auge de una nueva disciplina, nacida al amparo de la psicología social, la psicología ambiental, la que ha despertado algo el interés por la privacidad. Muestra del mismo es la publicación del monográfico que el Journal of Social Issues le dedicó en el 1977. Pero interés no es lo mismo que entusiasmo y diez años después la magna obra de psicología ambiental que compilan Stokols y Altman (1987) no dedica ningún capítulo al tema. Con todó, aparecen concepciones psicoambientales (Altman, 1975,1977 , Altman et al., 1981) que sitúan a la privacidad como concepto articulador del espacio personal, la territorialidad, el hacinamiento, en el ámbito de estudio del ambiente social (Darley y Gilbert, 1985).

Desde estas y otras perspectivas psicosociales han predominado dos concepciones de la privacidad: una incide en la idea de retirada, reclusión y evitación 
de la interacción (Bates, 1964; Shills, 1966) y la otra en la del control de la interacción y la libertad de elegir el momento y el lugar de la misma (Altman, 1975; Westin, 1967). Para los propósitos de este trabajo merece la pena recordar la definición de privacidad que da Bates (1964):

«El término puede definirse como el sentimiento que una persona tiene de que los demás deben ser excluidos de algo que sólo a él concierne, así como el reconocimiento de que los demás tienen derecho a hacer lo mismo» (p. 429).

Es curioso cómo también el concepto de comunidad se ha colado en la muestra psicosocial de la mano de otra reeiente disciplina, la psicología comunitaria, que, aunque de paternidad dudosa, ha encontrado en la psicología social sus mejores genes científicos. Hay que buscar en la obra de Tönnies (1979) «Comunidad y Asociación» el origen del interés que los psicólogos sociales tienen por este tema. Para este autor comunidad y asociación constituyen formas de agrupamiento que son fruto de la voluntad humana. Mientras que en la asociación hay una clase de contrato público que obliga al mantenimiento de la interdependencia entre los individuos; en la comunidad hay una especie de pulsión instintiva hacia la vida comunitaria. Se genera una interdependencia de carácter orgánico entre el individuo y la comunidad. Para Tönnies «la teoría de la comunidad parte del supuesto de la perfecta unidad de las voluntades humanas en tanto que condición original o natural que mantiene a pesar de su dispersión empírica» (1979, p. 33). Otros pensadores sociales han prestado su talento a la comprensión de la comunidad, pero por lo que se refiere a nuestra disciplina, es la irrupción de la psicología comunitaria en las dos últimas décadas la que sitúa a la comunidad en la diana de sus esfuerzos investigadores. La obra de Sarason (1974) «El sentido psicológico de comunidad» marca el inicio de una línea de investigación psicosocial que ha propuesto al sentido psicológico de comunidad como el concepto central de la psicología comunitaria (Sarason, 1986). También en este caso un monográfico de revista ha recogido el incipiente interés que el tema despierta entre los investigadores. Nos referimos al que le dedica el Journal of Community Psychology en sus números uno y cuatro del volumen 14 (1986) y en el que se realiza la más exhaustiva revisión del sentido de comunidad. Remitimos a este espléndido monográfico para una correcta visión de las distintas concepciones y definiciones que el tema ha sugerido a la ciencia social. Para nuestros fines vamos a quedarnos con la concepción de McMillan y Chavis (1986) para quienes el sentido de comunidad implica el sentimiento de pertenencia, de membrecía, de implicación personal. Implica también la influencia recíproca entre el individuo y la comunidad; la integración y la satisfacción de necesidades y la conexión y participación emocional.

Iniciábamos este trabajo con un planteamiento dualista del tema, que sólo hemos abandonado para abordar por separado estas dos medias naranjas que son la privacidad y la comunidad. Esta dualidad, de difícil reconocimiento en la mayoría de las ocasiones, plantea también una pregunta doble a la psicología social: ¿son comunidad y privacidad conceptos interdependientes?; ¿qué factores externos determinan la primacía de un sentimiento u otro?

La idea de un conflicto inherente a la relación entre privacidad y comunidad ha sido la contestación que la literatura científico-social ha proporcionado a la primera pregunta. Buen ejemplo es la afirmación de Slater (1974, p. 4): «la privacidad y la comunidad son necesidades antitéticas y no pueden ser maximizadas simultáneamente». Por su parte, Keyes (1973) advierte de la tendencia generalizada en la sociedad occidental de proteger más la privacidad que la 
comunidad. Y con ello se plantea la respuesta a la segunda pregunta. De nuevo Slater (1970) afirma que la actual supremacía de la privacidad sobre la comunidad no es debida, desde luego, a la existencia de una motivación humana fundamental, sino más bien a la reacción ante la pérdida de control sobre el ambiente. El hacinamiento, la sobreestimulación, la complejidad social son fẹnómenos característicos de las sociedades industriales y posindustriales que dificultan el control de los individuos sobre sus encuentros diarios. Un mecanismo puro de reactancia le empuja a enfatizar los aspectos más relacionados con la privacidad. Para Bookchin (1974) la complejidad urbana provoca en el individuo la pérdida percibida de control debido a la sobrecarga estimular, que a su vez le empuja a desear niveles altos de privacidad en un intento de reducir los inputs; reducción que le proporciona un mayor control. Esta idea no es nueva y ya Georg Simmel apuntaba que la existencia urbana exigía una distancia entre el individuo y su entorno social, «puesto que la pujante masificación y el desorden diversificado de la comunicación metropolitana sería simplemente insoportable sin esa distancia psicológica» (Simmel, 1896, p. 787).

Parece existir, pues, una estrecha relación entre el entorno del individuo y su sentimiento de comunidad y/o privacidad. Podría decirse que algunos entornos favorecen el aislamiento de las personas y otros su contacto. Son, en la terminología de Osmond (1957), los ambientes sociófugos y sociópetos, respectivamente. Hay ambientes que favorecen el sentido de pertenencia social y de comunidad y otros que engendran la desintegración y la alienación sociales (Little, 1987). Para Alexander (1967) la industrialización y su consecuencia más inmediata, la urbanización, desencadenan el repliegue del ser humano sobre sí mismo, como forma de afrontar el estrés generado por tales fenómenos contemporáneos. Llegado un momento - quizás histórico; quizás el nuestro- el individuo cree en su propia autosuficiencia e independencia de los demás. Deja de padecer «el hambre de comunidad», de sentir solidaridad, pertenencia e integración social. Las comunidades no pasan de ser «meros agregados o asociados político-administrativos» (García González, 1988), como advirtieron en la anterior postrimería Tönnies (1979). y Simmel (1986).

El contexto teórico que brevemente se dibuja más arriba nos sirve para intentar responder empíricamente a las preguntas abiertas antes. $\dot{A}$ tal fin presentamos dos investigaciones en las que poner a prueba la relación negativa entre privacidad y comunidad; así como explicitar algunas de las características individuales y sociales que determinan la supremacía de uno u otro sentimiento. Los dos trabajos siguen la estrategia de investigación que Fernández Dols (1990) denomina observación pasiva con modelos causales.

En la lógica que se diseña más arriba suponemos que un entorno sobrecargado de estímulos, como es el habitual en las grandes ciudades, influiría positivamente en el sentido de privacidad y negativamente en el de comunidad. Así, aquellos sujetos sometidos a una mayor exposición urbana (elevada densidad de población interior y exterior, ruido, suciedad, transportes públicos, restricciones espaciales) tendrán un menor sentido de comunidad y mayor de privacidad. Junto a estas condiciones físicas objetivas, nos pareció importante incluir en nuestros dos trabajos la apreciación subjetiva que tiene el individuo de su entorno y que,como es sabido, no siempre se corresponden todo lo razonablemente que se espera. Quizá el ejemplo más claro es el de la densidad de población y el hacinamiento (recuérdese la distinción de Stokols, 1972), que mantienen débiles relaciones, como han descubierto Gove y Hughess (1983) o West (1982). 
Así, en las dos investigaciones hemos incluido mediciones de los aspectos ambientales subjetivos, con la suposición de que una valoración positiva del entorno favorecerá los sentimientos comunitarios en detrimento de los de privacidad.

Otra variable que nos pareció relevante en la predicción de la privacidad y la comunidad fue el apoyo social. El apoyo social, que figura hoy día como una de las categorías psicosociales por excelencia (Blanco, 1988), dibuja un entorno social apto para las relaciones de solidaridad, intercambio mutuo, ayuda recíproca, preocupación por los demás, etc. Obviamente, un ser humano que disfrute de una buena red de relaciones que le proporcione un correcto apoyo social desarrollará más sentimientos de comunidad que de privacidad. Y esta es otra de las hipótesis que intentamos verificar y que tiene algún antecedente en el trabajo de Pretty (1990). Este autor ha comprobado que el sentido de comunidad está asociado con la posesión de buenas redes interpersonales y de apoyo social, en una investigación realizada con estudiantes de una residencia universitaria. Otro tanto puede decirse del trabajo de Glynn (1981) que pone de relieve la importancia del vecindario, de las conexiones que se mantiene con el mismo y de lo satisfactorio que resulta, en la determinación del sentido de comunidad y de privacidad.

Los dos conceptos que nos ocupan deben estar, obviamente, relacionados con las características de personalidad de los individuos. Pero sólo una de las posibles se estudió: la introversión. De mínima puede calificarse la investigación que relaciona estas variables. En el trabajo de Bowers (1979) se estableció que la necesidad de privacidad se relaciona sistemáticamente con las variables de personalidad que indican el deseo de reducir el contacto social. El hombre «privado y anticomunitario» descrito por este autor es una especie de ser patológico: es desconfiado, ordenado y meticuloso en los asuntos cotidianos, tiende al sedentarismo y a la actividad física televisada; se siente inferior a los demás, es reservado en sus relaciones sociales - ¿pero las tiene? - y le resulta difícil entablar una conversación; no es especialmente altruista y ejerce el desinterés por los demás; cree en lo nefasto de la influencia que tienen los demás sobre las cosas que le pasan. Sobra explicitar nuestra hipótesis con respecto a esta variable.

\section{METODOLOGIA}

\section{Muestra}

Un total de 339 sujetos participaron en la primera de las investigaciones, cuya edad media era de 20 años; de los que 253 eran mujeres, y 86, varones. Cursaban primero y segundo de Trabajo Social, 136; primero de Magisterio, 59; 91 eran de tercero de BUP; y 53 estudiaban FP-2.

Los 95 sujetos de la segunda investigación tenían una edad media de 34 años, y estudiaban en dos centros de educación para adultos de la provincia de Málaga - uno en la capital y otro en un pueblo-. Un total de 75 mujeres y 16 varones respondieron a las preguntas.

\section{Instrumentos de evaluación}

En los dos estudios se realizó un amplio cuestionario que contenía las preguntas correspondientes a los instrumentos de evaluación que pasamos a descri- 


\section{8}

bir ahora. Los sujetos debían responder en sendas hojas de respuestas. Los 95 sujetos del segundo estudio cumplimentaban sus respuestas en hojas preparadas para su procesamiento automático con una lectora óptica.

Para el análisis de las distintas subescalas hemos seguido las recomendaciones de Bollen (1986) de utilización de los modelos de ecuaciones simultáneas para la comprobación de hipótesis de constructo. Desde esta perspectiva se contrastâ un modelo teórico matemáticamente formalizable y los datos empíricos (Gómez Benito, 1986). Los resultados de este análisis se han obtenido a través del programa PC-Lisrel, desarrollado por Jöreskog y Sörbom (1986), utilizando la estimación por mínimos cuadrados no ponderados. La escasez de espacio impide la presentación de los principales resultados, que son considerablemente ajustados a las estructuras teóricas propuestas por los constructores de las escalas. En cualquier caso, estos datos psicométricos están a disposición del lector que los desee.

\section{Subescala de privacidad}

Veintisiete ítems que se agrupan en torno a seis dimensiones sirven para evaluar la privacidad. Son una adaptación de los elaborados por Pedersen (1979), que encontró una estructura subyacente a los mismos de seis factores: reserva, aislamiento, soledad, intimidad con la familia, intimidad con los amigos y anonimato. En líneas generales, estas seis dimensiones son indicadoras del control selectivo del acceso a sí mismo o al grupo en el que se mueve el individuo. Esta subescala se utilizó en los dos estudios.

\section{Subescala de contacto con vecinos y amigos}

Es una versión de una de las subescalas desarrolladas por Marshall (1974) y evalúa el deseo del individuo de no tener como vecinos a los amigos íntimos y no mantener muchas relaciones con los primeros. Esta variable está relacionada con el deseo de controlar el grado de autodescubrimiento ante los demás. Sólo se utilizó en el caso del primer estudio.

\section{Subescala de Individualismo-Comunalismo}

A propósito de este trabajo se han construido 18 ítems que pretendían explorar las ideas más o menos individualistas de los sujetos; la creencia de que el individuo es autosuficiente y de que la ligazón que mantiene con el resto de sus contemporáneos no es más que una pesada cadena. Esta variable se extiende desde un polo de individualismo hasta otro de comunalismo. Este instrumento no se utilizó en el segundo estudio.

\section{Subescalas de Comunidad}

También en este caso construimos una serie de preguntas cercanas al concepto de sentido psicológico de comunidad arriba mencionado: percepción de similitud con otras personas, interdependencia entre las mismas, reciprocidad, sentimiento de formar parte de una estructura social mayor, etc. Las preguntas de este cuestionario se agrupan en torno a cuatro variables: implicación con otras personas, ayuda y preocupación por ayudar a los demás, independencia y autosuficiencia, y competición con los demás en la mayoría de las actividades cotidianas. Se utilizó en ambos estudios. 


\section{Subescala de deseo de pertenencia grupal}

Es ésta una versión de la desarrollada por Turnbull (1979), en la que se prentendía evaluar el deseo del individuo de estar inmerso en una tupida tela de araña de relaciones con los demás en diferentes grupos. Usado sólo en la primera investigación.

\section{Evaluación subjetiva del entorno}

Hemos utilizado aquí el diferencial semántico. En el caso del primer estudio se utilizaron ocho pares de adjetivos que indicaban en qué medida consideraban los sujetos agradable su ciudad. Se corresponde, en parte, con la dimensión de agrado obtenida por Corraliza (1987) en su trabajo sobre la evaluación afectiva del ambiente.

En el segundo estudio hemos utilizado siete pares de adjetivos para evaluar la casa donde vive el sujeto y otros tantos para la evaluación de su barrio. Estos siete pares de adjetivos son indicadores del hacinamiento de cada uno de los dos ambientes.

\section{Subescalas sobre apoyo social}

Los resultados de nuestro trabajo otorgan validez a la estructura teórica defendida en la mayoría de las investigaciones realizadas desde la perspectiva funcional del apoyo social (Barrón, Lozano y Chacón, 1988); así como con las escalas desarrolladas por Cohen y colaboradores (1985), de los que son una versión las que nosotros utilizamos aquí. Apoyo emocional, informacional y tangible o instrumental son los tres factores que integran esta constructo. Este instrumento se usó en el primer estudio.

También utilizamos en el primer estudio las cuestiones de introversión del conocido cuestionario de Eysenck. Y de las respuestas obtenidas en una encuesta sobre las condiciones físicas objetivas en las que vivía el sujeto, construimos cuatro indicadores relacionados con la vida eminentemente urbana, y que hemos denominado urbanismo - sólo para el primer trabajo-. Tales indicadores son: Densidad de población en el barrio, densidad en la manzana/bloque, densidad en la casa y sobreestimulación urbana (ruidos, basuras, inseguridad, etc.). De forma similar en el segundo estudio construimos un solo indicador, al que denominamos densidad de población, y que une los distintos tipos de densidad a las que puede estar sometido el individuo.

\section{RESULTADOS}

Los resultados se obtuvieron a través del análisis de ecuaciones estructurales con el programa PC-Lisrel, ya mencionado, y estimaciones de máxima verosimilitud, en ambos casos. Los diagramas de vías de las figuras 1 y 2 muestran los principales resultados obtenidos en el primer y segundo estudio, respectivamente. Con respecto al primero de ellos, los diversos índices de bondad de ajuste revelan una considerable consistencia entre el modelo teórico propuesto y los datos empíricos. La probabilidad asociada al chi cuadrado es menor de 0.05 , lo cual implica un ajuste bajo. Sin embargo, son muchos los investigadores (v.g. Bentler y Bonnett, 1980) que son cautos a la hora de interpretar literalmente 
el significado de chi cuadrado, tan sensible al tamaño de la muestra. Por ello, siguiendo las recomendaciones de Bohrnstedt (1983), hemos establecido una ratio entre el valor de chi cuadrado y los grados de libertad del modelo (x2/gl). Cuanto más cercano a la unidad sea este resultado, mejor será el ajuste entre el modelo y los datos. En nuestro caso la ratio es igual a 1.77, no excesivamente lejano del resultado ideal. Los que aparecen en el cuadro también están muy cerca del ideal; de 1 para el índice de bondad de ajuste general (GFI) y el índice ajustado de bondad de ajuste general (AGFI) y el 0 para la raíz media cuadrada residual (RMSR).

Figura 1

Diagrama de vías del modelo causal correspondiente al primer estudio

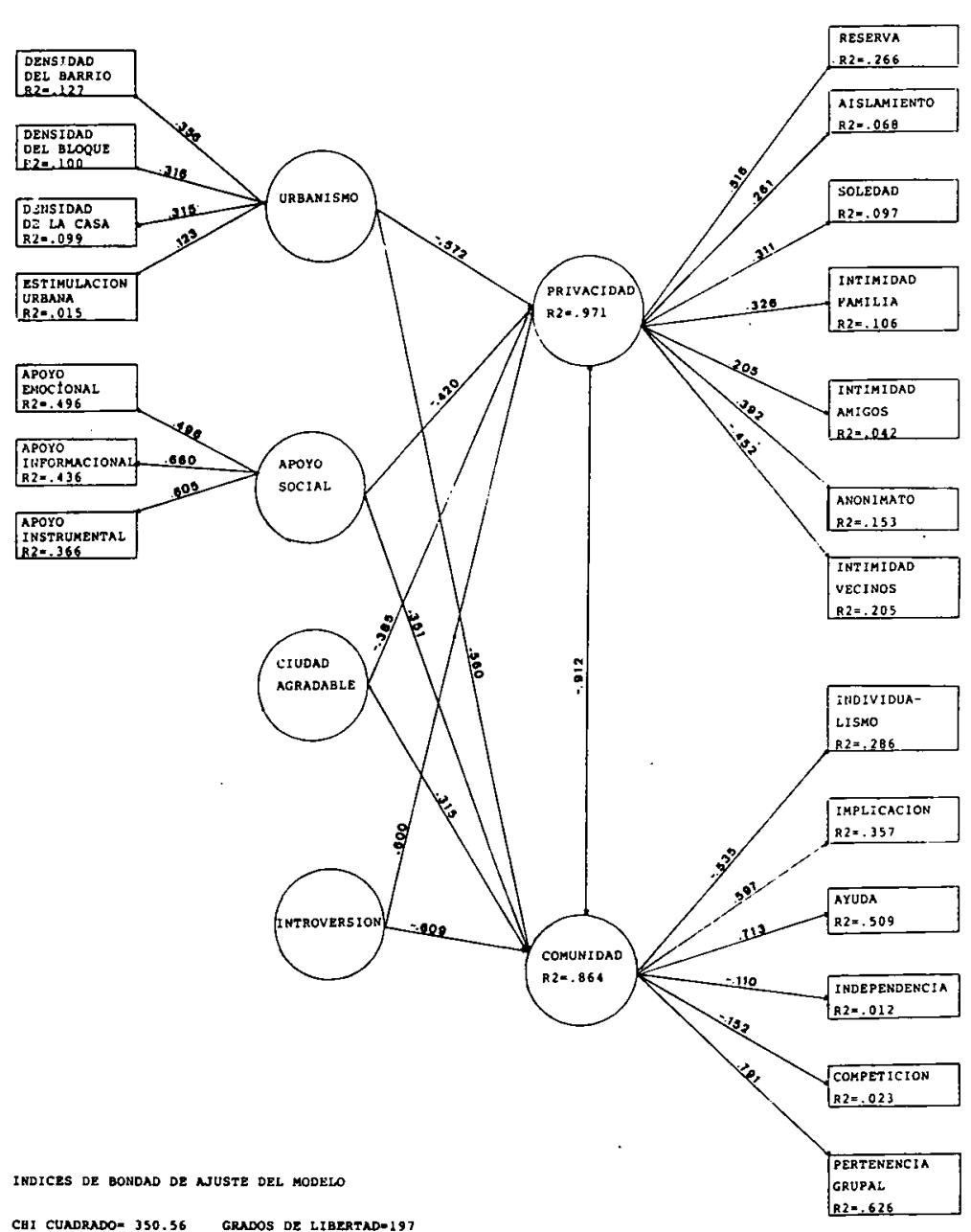




\section{1}

En lo referente a los coeficientes locales del modelo, lo primero a resaltar es la confirmada correlación negativa entre privacidad y comunidad $(\mathrm{r}=,-912)$. Ambos conceptos son considerados aquí como dos variables latentes endógenas, cada una de ellas con sus respectivos indicadores o variables directamente observadas. Para la privacidad los resultados indican una estructura en la que todas las variables, salvo la intimidad con los vecinos, «cargan» positiva y considerablemente en dicho constructo y todas con una probabilidad menor de 0.05 . Como cabía esperar la intimidad con los vecinos es contraria a un fuerte sentimiento de privacidad y «carga» negativamente. En la figura 1 pueden verse las correlaciones múltiples al cuadrado (R2) debajo de todas las variables observadas. Este valor puede interpretarse como un índice de la fiabilidad con que la variable mide el constructo. La variable latente comunidad ha sido medida a través de seis indicadores. Todos, menos independencia y competición con los demás, tienen coeficientes positivos, con probabilidades asociadas menores de 0.05 .

La primera sorpresa nos la proporciona la variable latente exógena urbanismo, definida primordialmente por indicadores de densidad de población, todos ellos positivos y con probabilidades también menores de 0.05 . Este constructo tiene una incidencia sobre privacidad y comunidad muy fuerte -las probabilidades de los parámetros son menores de 0.05 - pero de sentido contrario al que nosotros habíamos postulado. Así, cuanto mayores sean los estímulos urbanos y mayor la densidad de población, menor será el deseo de privacidad y mayor el sentido de comunidad. Más adelante intentaremos ocuparnos de estos resultados. El impacto del resto de los constructos exógenos sobre privacidad y comunidad confirman el patrón de relaciones causales que habíamos propuesto. El apoyo social, construido a partir de las tres variables observadas del diagrama, hace que los sujetos experimenten menos deseos de privacidad y más de comunidad. Estos efectos tienen el aval de probabilidades menores de 0.05 . También cuando los individuos valoran positivamente y consideran con agrado su ciudad disminuye la privacidad y aumenta el sentido de comunidad (para los dos $\mathrm{p}<0.05$ ). Los sujetos introvertidos, parece obvio, manifiestan más predilecciones por la privacidad y menos por la comunidad ( $p>0.05$ en ambos casos). Obsérvese en la figura 1 que el coeficiente de determinación (R2) que aparece bajo privacidad y comunidad es muy alto; .971 y .864, respectivamente, lo que es también un buen indicador de la bondad de los resultados.

No podemos decir lo mismo de los obtenidos en el segundo trabajo, que se ven en la figura 2. Si bien los índices de bondad de ajuste son similares a los anteriores, los parámetros locales son considerablemente más bajos. Por lo que respecta a los primeros, aunque aquí la probabilidad de chi cuadrado es mayor de 0.05 , tampoco puede hablarse de un ajuste elevado. Con la cautela precedente hemos calculado la ratio entre chi cuadrado y grados de libertad, arrojando un resultado de 1.36, que mejora al del primer estudio. El resto de los índices globales de ajuste, aunque débilmente, también mejoran.

Por lo que respecta a los resultados del modelo de medida para las variables latentes endógenas privacidad y comunidad, la tendencia de los mismos es idéntica al caso anterior, pero aquí hay dos indicadores de privacidad -intimidad con la familia e intimidad con los amigos - que tienen probabilidades mayores de 0.20 . Lo mismo sucede con comunidad, cuyo indicador competición «carga» débilmente aunque negativamente como esperábamos, siendo la probabilidad asociada también mayor de 0.20 . Puede apreciar el lector que en este estudio prescindimos de intimidad con los vecinos y de individualismo. 
Las variables exógenas de esta investigación no son las mismas - pero sí parecidas - que las anteriores. El hacinamiento es la única latente y viene correctamente definida por sus dos indicadores; los dos con parámetros positivos y con probabilidades menores a 0.05 . La densidad viene dada exclusivamente por una sola medida, que describimos más arriba. Y es ésta la variable que nos vuelve a sorprender aunque con menor intensidad que antes. De nuevo aparece el mismo patrón: a medida que aumenta la densidad de población aumenta la comunidad y disminuye la privacidad, si bien los coeficientes son pequeños y en el primer caso tiene una probabilidad mayor de $0.20 \mathrm{y}$ en el segundo se mue-

Figura 2

Diagrama de vias del modelo causal correspondiente al segundo estudio

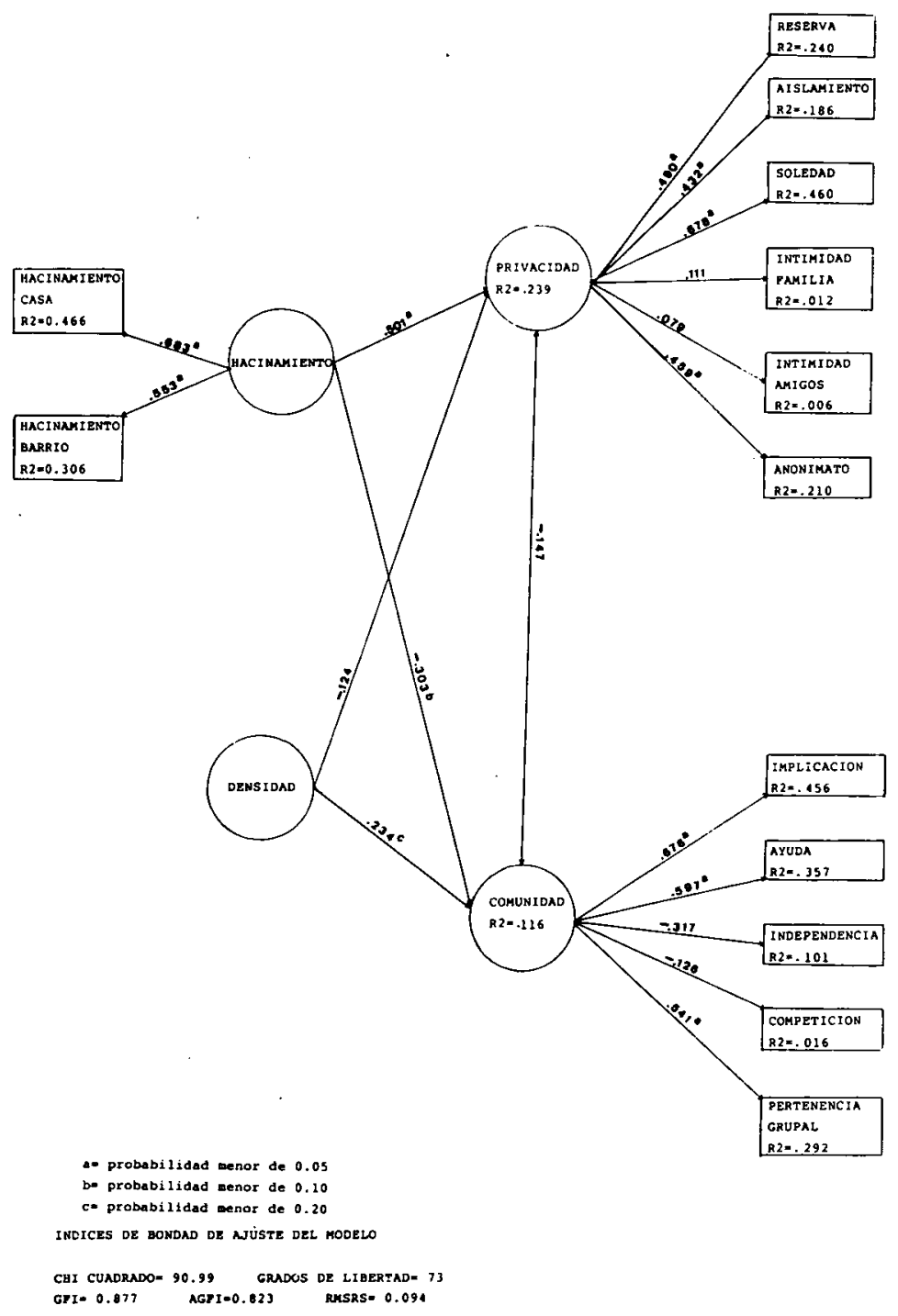


ve entre 0.20 y 0.10 . Pequeño es también el impacto del hacinamiento sobre nuestras dos variables, pero acorde con nuestras expectativas: hace aumentar el deseo de privacidad $(p<0.05)$ y disminuir el de comunidad $(p>0.10-p<0.20)$. Finalizamos este apartado con otro resultado que confirma tenuemente nuestro modelo; la correlación entre privacidad y comunidad es negativa ( $r=-.147)$.

\section{DISCUSION}

Los resultados apoyan la vieja idea de que comunidad y privacidad son antitéticas y que generalmente la maximización de una, significa el detrimento de la otra. Quizá también podríamos afirmar —en un intento de cerrar la segunda pregunta que formulamos más arriba - que un entorno hostil favorece la reclusión del individuo y erosiona el sentido de comunidad. Pero esto sería solamente cierto para las variables subjetivas que delimitan ese entorno hostil: ciudad desagradable, hacinamiento, falta de apoyo social. Como vimos, los indicadores objetivos no se portaron bien con nuestros planteamientos teóricos, y les dieron totalmente la vuelta: la densidad de población alta y el exceso de urbanismo favorecen el sentido de comunidad y minimizan el de privacidad.

Este resultado pone sobre el tapete la discusión sobre la utilización de indicadores subjetivos versus indicadores objetivos a la hora de estudiar variables psicosociales. Refiriéndose al concepto de calidad de vida, Blanco Abarca (1985) ya nos avisa de los problemas que acarrea, en ese campo, la utilización de criterios puramente subjetivos en la evaluación de la calidad de vida. Dice este autor: «De esta manera es muy fácil seguir sospechando que se puede ser perfectamente feliz en medio de la pobreza y de la ignorancia y que la riqueza, en último término, da muchos disgustos y no menos quebraderos de cabeza» (1985, p. 179). Podríamos hacer una interpretación de nuestros resultados en términos parecidos. Esta distinción entre los criterios objetivos y los psicológicos y subjetivos la realiza también Stokols (1972) para diferenciar densidad de población y hacinamiento. Se utiliza el primer término para referirse estrictamente a los aspectos físicos y/o espaciales de la situación. Puede ser operacionalizado mediante el número de personas por unidad de espacio disponible. Por el contrario, el hacinamiento alude a un estado subjetivo, a la experiencia psicológica originada por una demanda de espacio por parte del sujeto que excede del disponible. Mencionábamos más arriba la escasa relación empírica entre ambos conceptos. En nuestro caso - aunque no aparece en las figuras por razones de claridad espacial- estas relaciones no son altas pero tampoco escasas. En el primer estudio la correlación entre urbanismo y agrado de la ciudad es de .406; en el segundo, la existente entre densidad y hacinamiento es .218. En ambos casos era esperable el sentido de la correlación aunque la fuerza de la misma debería ser mayor. Si las variables no son intercambiables, sus respectivos efectos tampoco tienen por qué serlo. Las condiciones físicas objetivas necesitan pasar por «los siempre oscuros dominios de la percepción y valoración personales» (Blanco, 1985, p. 178) para que pasen a ser consideradas como positivas o negativas; a ser un fenómeno psicológico. En nuestros dos estudios, la experiencia psicológica negativa de su entorno -y no éste por sí mismo- es la que hace que los sujetos se retiren del mundanal ruido y sigan esa escondida senda de sabios, que dice el poeta. 
¿Por qué las condiciones físicas tienen una incidencia contraria? Permítasenos hacer un poco de psicología diferencial para intentar responder a esta pregunta. Recuérdese que la mayoría de los sujetos de ambos estudios eran mujeres. En un trabajo experimental, Epstein y Karlin (1975) formaron grupos de seis personas del mismo sexo que se asignaron a salas experimentales de tamaños distintos y evaluaron los sentimientos que manifestaban los sujetos hacia el resto de los miembros del grupo. Las actitudes negativas hacia los demás compañeros en la condición de alta densidad sólo se dieron en los varones. Las mujeres, por el contrario, adoptaron una actitud amistosa en esa condición. Un resultado similar es el encontrado por Ross y colaboradores (1973). Quizá hombres y mujeres, dicen estos autores, tienen experiencias parecidas en condiciones de alta densidad de población, pero socialmente el hecho de que las mujeres puedan comunicarse sus sentimientos y tensiones, y esté mal visto en los hombres, hace que aumente entre ellas el sentimiento de ayuda y de comprensión.

Es probable que otra explicación del resultado contradictorio que estamos analizando, se encuentre en los trabajos de Freedman (1975) que establecen una interacción entre la propia densidad y la situación más o menos positiva en la que se encuentra el individuo. Quizá nosotros deberíamos tener en cuenta no sólo los efectos aditivos de estas variables, sino también los debidos a su interacción. Ello es particularmente complicado de realizar desde la metodología que hemos utilizado (ver Bollen, 1989), máxime cuando los términos involucrados en la oportuna operación matemática son variables latentes. Aun así futuros trabajos deberían abordar este asunto. Y no vamos a insistir más en la búsqueda de explicaciones a unos resultados que no esperábamos.

El resto es coherente - unas veces más y otras menos- con el modelo teó. rico postulado. Los resultados referentes al apoyo social sostienen la norma de reciprocidad (Antonucci y Jackson, 1989), por la cual el individuo efectúa una especie de cálculo entre los haberes y el debe de apoyo, en un intento de mantener el equilibrio. Nuestros sujetos desarrollan un mayor sentido de comunidad y menor de privacidad cuando están inmersos en una red de relaciones sociales satisfactorias, proveedoras de un buen apoyo social. Y sucede lo contrario cuando predomina en ellos la introversión.

Finalizamos ya con el deseo de que ulteriores trabajos ayuden a cerrar más los interrogantes que planteamos arriba y a clausurar aquellos que suscita esta investigación. Pero ahora nosotros debemos abandonar el refugio privado de la pantalla de ordenador y salir a la comunidad de la página impresa. Al fin y al cabo este fin de siglo todavía nos permite gozar de ambas experiencias.

\section{Referencias}

AleXander, C.A. 1976. The city as a mechanism for sustaining human contact, en W.R. Ewald, Jr., Environment for man: The next 50 years, Bloomington: Indiana University Press.

Altman, I. 1975. The environment and social behavior, Monterrey: Brooks/Cole Publishing Company.

Altman, I. 1977. Privacy regulation: culturally universal or culturally specific?, Joumal of Social Issues, 33, 66-84.

Altman, I.; Vinsel, A. y Brown, B. 1981. Dialectic conceptions in social psychology: An application to social penetration and privacy regulation, Advances in Experimental Social Psychology, 14, 107-159. 
AntonucCI, T. C., y JACKson, J.S. 1989. Apoyo social, eficacia interpersonal y salud: Una perspectiva del transcurso de la vida, en L. L. Carstensen y B. A. Edelstein, Gerontologia clínica. Intervención psicológica y social, Barcelona: Martínez Roca.

Barron, A.; Lozano, P., y Chacón, F. 1988. Autoayuda y apoyo social, en A. Martín, F. Chacón y M. Martínez, Psicología Comunitaria, Madrid: Visor.

Bates, A. P. 1964. Privacy: A useful concept?, Social Forces, 42, 429-434.

BENTLER, P. M. Y BonNetr, D. G. 1980. Significance tests and goodnes of fit in the analysis of covariance structures, Psychological Bulletin, 88, 588-606.

Blanco Abarca, A. 1985. La calidad de vida: supuestos psicosociales, en J. F. Morales, A. Blanco, C. Huici y J. M. Fernández, Psicologia social aplicada, Bilbao: Desclée de Brouwer.

Blanco AbarCa, A. 1988. La psicología comunitaria, ¿una nueva utopía para el final del siglo XX?, en A. Martín, F. Chacón y M. Martínez, Psicología Comunitaria, Madrid: Visor.

Bohrnstedt, G. W. 1983. Measurement, en P. Rossi, J. Wright y A.'Anderson (eds.), Handbook of survey research, Nueva York: Academic Press.

Bollen, K. A. 1989. Structural equations with latent variables, Nueva York: John Wiley \& Sons.

Bookchin, M. 1974. The limits of the city, Nueva York: Harper Colophon.

BWERs, J. K. 1979. Privacy, territoriality, personal space and personality, Environment design research association, 10, 51-60.

Cohen, Sh., Mermelstein, R.; Kamarck, T. y Kaplan, R. M. 1985. Measuring the functional components of social support, en I. G. Sarason y B. R. Sarason. Social support: theory, research and applications, Dordrecht Martinus Nijhoff Publishers.

Corraliza, J. A. 1987. La experiencia del ambiente. Percepción y significado del medio construido, Madrid: Tecnos.

DARLEY, J. M., y GILBERT, D. T. 1985. Social psychological aspects of environmental psychology, en G. Lindzey y E. Aronson, The bandbook of social psychology, Nueva York: Random House.

Epstein, J. y KarLin, R. 1975. Effects of acute experimental crowding, Joumal of Applied Social Psychology, 5, 34-53.

Esser, A. H. y Greenbie, H. 1979. Design for communality and privacy, Nueva York: Plenum Press.

Fernández Dols,J. M. 1990. Patrones para el diseño de la Psicología Social, Madrid: Morata.

Freedman, J. L. 1975. Crowding and bebavior, San Francisco: W. F. Freeman and Company.

García GonzÁlez,J. A. 1988. Cuestiones y modelos teóricos en Psicología Comunitaria, en A. Martín, F. Chacón y M. Martínez, Psicología Comunitaria, Madrid: Visor.

GlynN, TH. J. 1981. Psychological sense of community: measurement and application, $\mathrm{Hu}$ man Relations, 34, 789-818.

Gómez Benito, J. 1986. Los modelos causales como metodología de validez de constructo, Barcelona: Alamex.

Gove, W. R. Y Hughes, M. 1983. Overcrowding in the housebold, Nueva York: Academic Press.

Kelvin, P. 1973. A social-psychological examination of privacy, British Joumal of Social and Clinical Psychology, 12, 249.261.

KEYES, R. 1973. We, the lonely people: Searching for community, Nueva York: Harper and Row.

Kimmel, A. J. 1988. Ethics and values in applied social research, Newbury Park: Sage Publications.

Jöresкog, K. G. y Sörвом, D. 1986. Lisrel VI: Analysis of linear structural relationships by maximun likelibood, instrumental variables and least squares methods, Uppsala: University of Uppsala.

LitrLe, B. R. 1987. Personality and the environment, en D. Stokols e I. Altman, Handbook of environmental psychology, Nueva York: John Wiley \& Sons.

Marshall, N. 1974. Dimensions of privacy preferences, Multivariate Bebavioral Research, 9, 255-272.

Mcmillan, D. W. y Chavis, D. M. 1986. Sense of community: A definition and theory, Journal of Community Psychology, 14, 6-23.

Osmond, H. 1957. Function as the basis of psychiatric ward design, Mental Hospitals, 8, 23-29.

Pedersen, D. H. 1979. Dimensions of privacy, Perceptual and Motor Skill, 48, 1.291-1.297.

Pretty, G. M. H. 1990. Relating psychological sense of community to social climate characteristics, Journal of Communty Psychology, 18, 60-65.

Ross, M.; Layton, B.; Erickson, B., y Schopler, J. 1973. Affect, facial regard and reactions to crowding, Joumal of Personality and Social Psychology, 28, 69-76. 
Sarason, S. B. 1974. The psychological sense of community, San Francisco: Jossey-Bass.

SARAsOn, S. B. 1986. Commentary: The emergence of a conceptual center, Journal of Community Psychology, 14, 405-407.

SHilss, E. 1966. Privacy: Its constitution and vicissitudes, Law and Contemporary Problems, $31,281-305$.

Simmel, G. 1896. Soziologische asthetik, Die Zukunfit, 17, 204-216.

Simmel, G. 1986. El individuo y la libertad. Ensayos de crítica de la cultura, Barcelona: Península.

SLATER, P. 1970. The pursuit of loneliness: American culture and the breaking point, Boston: Beacon Press.

Slater, P. 1974. Earthwalk, Nueva York: Anchor Press.

STOKOLS, D. 1972. On the distinction between density and crowding: some implications for further research, Psychological Review, 79, 275-277.

Stokols, D., y Altman, I. 1987. Handbook of environmental psycbology, Nueva York: John Wiley \& Sons.

Tönnies, F. 1979. Comunidad y asociación, Barcelona: Península.

Turnbull, A. A. Privacy, community and activity space. A relational exploration, en A. H.

Esser y B. B. Greenbie, op. cit.

WEST, P. C. 1982. Effects of user behavior on the perception of crowding in backcountry forest recreation, Forest Science, 28, 95-105.

Westin, A. F. 1976. Privacy and freedom, Nueva York: Atheneum. 